Nota científica

\title{
Subestimación de la abundancia del pepino café Isostichopus fuscus (Holothuroidea: Echinodermata) en muestreos diurnos con respecto a nocturnos en el golfo de California
}

\author{
Underestimation of the abundance of brown sea cucumber Isostichopus fuscus (Holothuroidea: \\ Echinodermata) in daylight compared to nighttime surveys in the Gulf of California \\ Héctor Reyes-Bonilla $^{a}$, Georgina Ramírez-Ortiz ${ }^{\mathrm{b}}$, María Dinorah Herrero-Pérezrul ${ }^{\mathrm{c}}$ \\ y Luis E. Calderon-Aguilera ${ }^{\mathrm{b}, *}$ \\ ${ }^{a}$ Universidad Autónoma de Baja California Sur, Departamento Académico de Biología Marina, Carretera al sur Km 5.5, Col. El Mezquitito, 23080, \\ La Paz, Baja California Sur, México \\ ${ }^{\mathrm{b}}$ Centro de Investigación Científica y de Educación Superior de Ensenada, Carretera Ensenada-Tijuana 3918, Zona Playitas, 22860, Ensenada, \\ Baja California, México \\ ${ }^{\mathrm{c}}$ Centro Interdisciplinario de Ciencias Marinas, Instituto Politécnico Nacional, Departamento de Pesquerías y Biología Marina, Av. Instituto Politécnico \\ Nacional s/n, Col. Playa Palo de Santa Rita, Apartado postal 592, 23096, La Paz, Baja California Sur, México
}

Recibido el 1 de octubre de 2015; aceptado el 21 de enero de 2016

Disponible en Internet el 27 de mayo de 2016

\begin{abstract}
Resumen
El pepino café Isostichopus fuscus es una especie de alto valor comercial y en México está bajo protección federal. La pesquería se desarrolla en la península de Baja California, siguiendo reglas de manejo determinadas en Mesas Técnicas mediante acuerdos entre agencias de gobierno, científicos y pescadores organizados. Uno de ellos es que las evaluaciones de abundancia deben hacerse durante el día, cuando el pepino se oculta en oquedades, arrojando un valor inferior, pero no se ha comprobado. El objetivo del presente trabajo fue comparar la abundancia del pepino café en censos conducidos en transectos de $100 \times 2 \mathrm{~m}$, fijos al fondo y realizados durante el día y la noche del mismo día. Los resultados indican que hubo significativamente más pepinos observados en horas de oscuridad (promedio \pm error típico $=3.714 \pm 0.075)$ que de luz (1.943 \pm 0.045$)$, y que además es posible corregir la abundancia por transecto en el día con una ecuación lineal (pendiente $=1.43 ;$ ordenada $=1.18$ ). La diferencia de cuota potencial calculada con los censos de día es aproximadamente un $15 \%$ menor a la que se obtendría en conteos nocturnos. Se concluye que la medida actual debe ser exitosa para un manejo precautorio del recurso.

Derechos Reservados (C) 2016 Universidad Nacional Autónoma de México, Instituto de Biología. Este es un artículo de acceso abierto distribuido bajo los términos de la Licencia Creative Commons CC BY-NC-ND 4.0.
\end{abstract}

Palabras clave: Manejo precautorio de recursos pesqueros; Holoturoideos; Factor de corrección; Censos visuales; Especie sujeta a protección especial; Bahía de Loreto

\section{Abstract}

The brown sea cucumber Isostichopus fuscus, is a holothurid with high commercial value. In Mexico it is federally protected, and the fishery in the Baja California Peninsula follows management actions established by technical committees, on the basis of agreements among government agencies, scientists and organized fishermen. One of these agreements is that evaluations of its abundance must be done during the day, as it has been suggested that during daylight hours the organisms hide inside holes, resulting on lower values, but this has not been demonstrated yet. Thus the objective of this study was to compare the abundance in census conducted inside fixed transects of $100 \mathrm{x} 2 \mathrm{~m}$, and surveyed at day and night of

\footnotetext{
* Autor para correspondencia.

Correo electrónico: leca@cicese.mx (L.E. Calderon-Aguilera).

La revisión por pares es responsabilidad de la Universidad Nacional Autónoma de México.
} 
the same day. The results show that there were significantly more sea cucumbers seen during dark hours (mean \pm standard error $3.714 \pm 0.075)$ than during light time $(1.943 \pm 0.045$ per census); daylight estimated abundance can be corrected by a linear equation (slope $=1.43$; ordinate $=1.18)$. Therefore, the quota estimated from censuses conducted during daylight is $15 \%$ lower than those that would be obtained at night. In conclusion, daylight censuses work as a precautionary management strategy of this species.

All Rights Reserved (C 2016 Universidad Nacional Autónoma de México, Instituto de Biología. This is an open access item distributed under the Creative Commons CC License BY-NC-ND 4.0.

Keywords: Precautionary fisheries management; Holothurian; Correction factor; Visual census; Species under special protection; Loreto Bay

El pepino café, Isostichopus fuscus (Ludwig, 1875) es una holoturia que se distribuye desde el golfo de California, México, hasta la costa de Perú, incluyendo islas oceánicas como las Revillagigedo y las Galápagos (Purcell, Smyn y Conand, 2012). Esta especie se captura comercialmente en varios países de América Latina —en especial Ecuador y México-, ya que alcanza precios superiores a 1,000 dólares americanos por kilo en el mercado de países orientales hacia donde se exporta su producción total (Purcell et al., 2012).

En México, el pepino café presenta densidades poblacionales altas únicamente en el golfo de California, donde actualmente es motivo de una pesquería bien desarrollada (Herrero-Pérezrul y Chávez, 2005). Históricamente, la especie comenzó a ser capturada para su venta desde finales de la década de 1980 y llegó a un auge a principios de 1990; sin embargo, la carencia de regulaciones llevó a un descontrol en las actividades pesqueras que dio como resultado la baja sensible en el tamaño de las poblaciones (Fajardo-León y Vélez-Barajas, 1996). Como consecuencia, el Gobierno Federal de México dispuso a I. fuscus dentro de la Norma Oficial Mexicana Nom-059-Ecol-1994, bajo la categoría de especie en riesgo (Semarnap, 1994). Inicialmente, se impuso una veda total a la pesca a partir de 1994, pero esta fue levantada en 2002, año a partir del cual la pesca legal se condujo dentro del régimen de unidades de manejo ambiental y, posteriormente, como predios de aprovechamiento (Semarnat, 2010). Al estar listada en la NOM, hoy en día por ley los permisos de pesca son otorgados por la Dirección General de Vida Silvestre de la Secretaría de Medio Ambiente y Recursos Naturales (Semarnat), bajo recomendación de las mesas técnicas del pepino de mar - conformadas por investigadores, pescadores, técnicos de cooperativas y representantes de dependencias gubernamentales-, las cuales han sido establecidas exclusivamente en los estados de Baja California y Baja California Sur.

Actualmente, el pepino café se encuentra bajo la categoría de «especie sujeta a protección especial» en la Nom-059-Semarnat2010 (Semarnat, 2010). Debido a su relevancia económica, el gobierno federal y el de los estados de Baja California y Baja California Sur, donde se lleva a cabo la explotación del recurso, han tratado de aplicar regulaciones, como, por ejemplo, una veda de junio a septiembre, aunque en algunos casos dichos controles se basan solo en acuerdos mutuos entre autoridades y asociaciones pesqueras, ya que a la fecha se carece de un programa de manejo del recurso que haya sido publicado oficialmente por la Semarnat. Entre las reglas pactadas dentro de la regulación del pepino café se encuentran los límites en las tasas de aprovechamiento determinados sobre la base de las evaluaciones in situ, la temporada de pesca - que normalmente se desarrolla de octubre a mayo-, la talla mínima de captura $-20 \mathrm{~cm}$ o $370 \mathrm{~g}$ por individuo-, así como la convención de que los permisos de pesca sean anuales y se otorguen preferentemente a los pescadores organizados con historial de aprovechamiento del recurso (Herrero-Pérezrul y Chávez, 2005).

Uno de los acuerdos clave de las mesas técnicas es que la captura del pepino café se debe llevar a cabo durante el día y tal decisión se tomó ya que, por experiencia de representantes de gobierno, académicos y pescadores, se sabe que las abundancias estimadas de I. fuscus son mayores si los censos se conducen en la noche, ya que aparentemente los organismos salen a alimentarse en áreas abiertas y lejos de sus escondites diurnos (Gutiérrez-García, 1999). De este modo, los censos llevados a cabo en las horas de luz subestiman la abundancia y por ello se usan como una medida precautoria para el manejo de la especie. A pesar de lo anterior, el sesgo que representa esta medida no ha sido cuantificado y el dato es importante como una medida de manejo. Tomando en cuenta esta situación, los objetivos del presente trabajo fueron comparar la diferencia entre la abundancia estimada del pepino café en conteos conducidos exactamente en los mismos transectos durante el día y la noche, para posteriormente usar esa información con el fin de proporcionar un factor de corrección útil para apoyar el manejo del recurso pepino café. Este es el primer trabajo en su tipo y es importante porque aunque empíricamente se sabe que visualmente hay menor abundancia durante el día, no se sabe en qué proporción, por lo que es necesario cuantificar esa diferencia.

El estudio se llevó a cabo entre los días 12 y 19 de octubre del 2008, en la localidad de El Candelero de la isla Coronado, situada al norte de la bahía de Loreto (coordenadas aproximadas $26^{\circ} 08^{\prime} \mathrm{N}, 111^{\circ} 16^{\prime} \mathrm{O}$ ), en el margen suroriental de la península de Baja California. Para determinar los cambios en la abundancia de individuos de I. fuscus durante las horas de luz y de oscuridad se llegaba al punto de muestreo durante la tarde, entre las 16:00 y 17:00, al menos $2 \mathrm{~h}$ antes de la puesta del sol. Mediante buceo autónomo se fijaban entre 8 y 12 flexómetros de $50 \mathrm{~m}$ de largo, amarrados en sus extremos a estacas clavadas en el fondo a profundidades de entre 3 y $16 \mathrm{~m}$. Una vez emplazados, se llevaron a cabo censos de individuos por medio de la revisión de una banda de $2 \mathrm{~m}$ de ancho paralela al flexómetro, por lo que cada transecto tenía $50 \times 2=100 \mathrm{~m}^{2}$ de área, en la cual se buscaron organismos en áreas abiertas o dentro de oquedades como bases de corales o huecos en la roca, como lo indican Herrero-Pérezrul, Reyes-Bonilla, García-Domínguez y Cintra-Buenrostro (1999). Posteriormente el mismo día, se dejaban pasar al menos $3 \mathrm{~h}$ para 
que, en oscuridad y ayudados de lámparas de buceo, se repitieran los censos exactamente en las mismas líneas de transecto y con idéntica metodología de conteo. En total, el procedimiento se repitió en 70 transectos recorridos tanto en el día como en la noche.

Los datos generados en campo correspondieron al número de pepinos vistos por transecto de $100 \mathrm{~m}^{2} \mathrm{y}$ fueron comparados con pruebas $t$ de Student, tomando el día y la noche como factores de prueba. Se usó un nivel de confianza del $95 \%$ y como los datos no fueron homoscedásticos se aplicó la prueba $t$ en su modalidad de varianzas desiguales (Zar, 2009). Posteriormente, se tomaron los datos del censo de día como variable independiente y los del censo nocturno como dependiente, y se utilizó una regresión lineal simple para detectar la relación entre ambos conteos. La regresión fue calculada por métodos iterativos —algoritmo de Newton-, usando la herramienta Solver de EXCEL ${ }^{\mathrm{TM}}$, con la finalidad de evitar el problema de la falta de homogeneidad de varianzas mencionado con anterioridad, lo cual hace impreciso el valor calculado de los estimadores si este se obtiene por métodos tradicionales de mínimos cuadrados (Weisberg, 2013).

La prueba $t$ de Student denotó diferencias significativas en el número de individuos contados en el día y la noche $(t=2.405, p=0.018, N=70)$, encontrando que los valores de abundancia fueron superiores en los censos nocturnos (conteos de día $=1.943 \pm 0.045$; conteos de noche $=3.714 \pm 0.075$; promedio \pm error típico). Por otra parte, la regresión lineal entre los censos diurnos y nocturnos (fig. 1) fue significativa $\left(r^{2}=0.5459\right.$. $\left.\mathrm{F}_{1.68}=81.761, p<0.0001\right)$, mostrando que durante la noche se observaron más organismos en la misma área revisada que durante el día. La tasa de cambio - es decir, la pendiente de la recta - indica que por cada individuo que se censa durante el día, existen 1.17 organismos en un área de censo de $100 \mathrm{~m}^{2}$; a esta cifra además debe adicionarse la ordenada al origen de la recta, que equivale a 1.43 individuos.

A manera de ejemplo, en la tabla 1 se simulan las diferencias que habría tanto en número de pepinos como en biomasa dependiendo de si los censos se hicieran bajo condiciones de luz o de oscuridad. Los valores de la primera columna son ficticios, pero se basan en ejemplos de cuotas normales otorgadas a las organizaciones de pescadores que manejan los predios, por sugerencia de las mesas técnicas. Como se observa, considerando una cuota típica de entre 10,000 y 500,000 individuos, la diferencia en el número de organismos estimado de día y noche

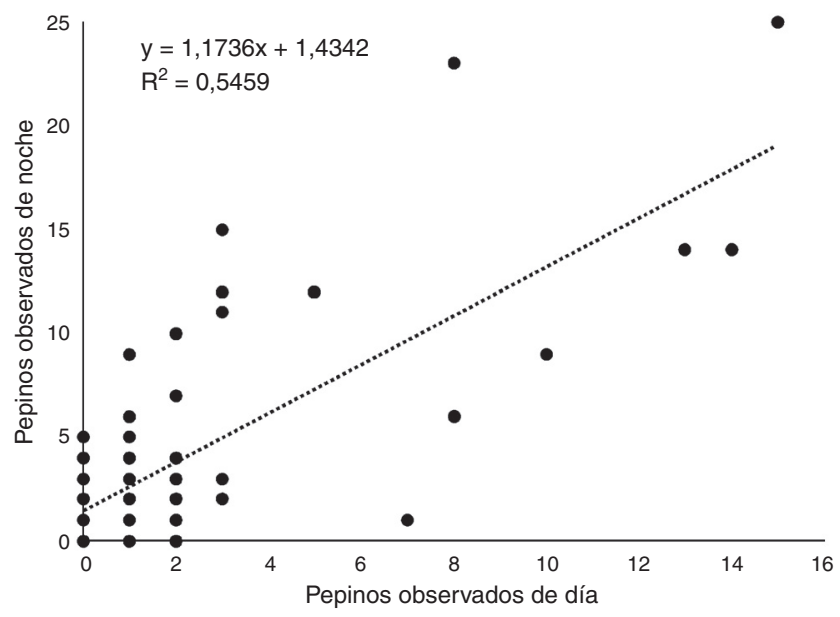

Figura 1. Regresión lineal simple entre el número de individuos del pepino café Isostitchopus fuscus observados de día y de noche en 70 transectos de $100 \mathrm{~m}^{2}$, efectuados en la bahía de Loreto, golfo de California. Los valores de los coeficientes de la regresión fueron: ordenada al origen $=1.4342 \pm 0.4925$ $(p=0.0049)$ y pendiente $=1.1736 \pm 0.1298(p<0.0001)$.

es de entre 17,000 y 86,000 individuos, aproximadamente equivalentes a entre 5.6 y 28.2 ton. Así, los estimadores de captura potencial basados en evaluaciones de la abundancia tomadas de día arrojan un $15 \%$ menos del valor esperado de la abundancia del stock, lo que sería el beneficio neto de la medida de manejo ya en aplicación.

Como conclusión, en este trabajo se confirma la suposición de que los conteos de individuos del pepino café efectuados de noche generan valores más altos que los realizados durante el día y apoya su uso como un mecanismo de manejo del recurso. La abundancia estimada en horas diurnas es aproximadamente menor en un $15 \%$ a las abundancias potenciales, por lo que las evaluaciones en horas de luz dan lugar a la solicitud de cuotas más bajas que las reales, y por ello la medida puede tomarse como exitosa para un manejo precautorio del recurso. Además, presentamos una ecuación para ajustar los valores esperados de abundancia de la especie, que puede usarse por las autoridades como un indicador de la condición real de las poblaciones. Si bien este factor de corrección pudiera variar en función de condiciones muy particulares del sitio y de la época del año, es un buen estimador para fines de manejo.

Tabla 1

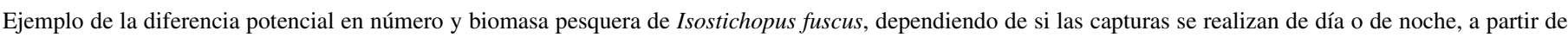

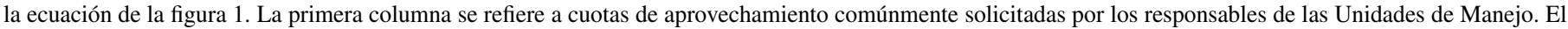

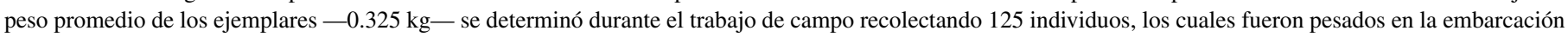
con una pesola con precisión de $1 \mathrm{~g}$..

\begin{tabular}{|c|c|c|c|c|c|}
\hline $\begin{array}{l}\text { Número de pepinos } \\
\text { otorgados como cuota } \\
\text { (censados de día) }\end{array}$ & $\begin{array}{l}\text { Número de individuos } \\
\text { potencialmente presentes } \\
\text { (censados de noche) }\end{array}$ & $\begin{array}{l}\text { Diferencia en } \\
\text { número entre } \\
\text { noche y día }\end{array}$ & $\begin{array}{l}\text { Biomasa de captura (en } \mathrm{kg} \text { ) } \\
\text { calculada sobre la base de } \\
\text { la cuota (censos de día y } \\
\text { considerando un promedio } \\
\text { de } 0.325 \mathrm{~kg} \text { por individuo) }\end{array}$ & $\begin{array}{l}\text { Biomasa de captura (en } \mathrm{kg} \text { ) } \\
\text { potencialmente presente } \\
\text { (censos de noche y } \\
\text { considerando un promedio } \\
\text { de } 0.325 \mathrm{~kg} \text { por individuo) }\end{array}$ & $\begin{array}{l}\text { Diferencia en } \\
\text { biomasa entre } \\
\text { noche y día }(\mathrm{kg})\end{array}$ \\
\hline 10,000 & $11,737.4$ & $1,737.4$ & 3,250 & $3,814.7$ & 564.7 \\
\hline 100,000 & $117,361.4$ & $17,361.4$ & 32,500 & $38,142.5$ & $5,642.5$ \\
\hline 300,000 & $352,081.4$ & $52,081.4$ & 97,500 & $114,426.5$ & $16,926.5$ \\
\hline 500,000 & $586,501.4$ & $86,501.4$ & 162,500 & $190,710.5$ & $28,210.5$ \\
\hline
\end{tabular}


El estudio se llevó a cabo con fondos de la Comisión Nacional de Áreas Naturales Protegidas (PROCODES, 2008, a HRB), y Sagarpa-Conacyt (2013-225235, a LECA). Se agradece a Verónica Castañeda, Aracely Rojas, Nuria Torrejón, Arturo Ayala, Lázaro Cadena, Andrés González y Sergio Scarry González, su ayuda para la realización de los censos.

\section{Referencias}

Fajardo-León, M. C. y Vélez-Barajas, A. (1996). Pesquería del pepino de mar. En M. C. Casas-Valdez y G. Ponce-Díaz (Eds.), Estudio del potencial pesquero y acuícola de Baja California Sur. (2) (pp. 152-165). La Paz, Baja California Sur: Semarnap/Cicimar.

Herrero-Pérezrul, M. D. y Chávez, E. A. (2005). Optimum fishing strategies for Isostichopus fuscus (Echinodermata: Holothuroidea) in the Gulf of California, Mexico. Revista de Biología Tropical, 53, 357-366.

Herrero-Pérezrul, M. D., Reyes-Bonilla, H., García-Domínguez, F. y CintraBuenrostro, C. E. (1999). Reproduction and growth of Isostichopus fuscus
(Echinodermata: Holothuroidea) in the southern Gulf of California, Mexico. Marine Biology, 135, 521-532.

Gutiérrez-García, A. (1999). Potential culture of sea cucumber in Mexico. Bêche de Mer Information Bulletin, 11, 26-29.

Purcell, S. W., Smyn, Y. y Conand, C. (2012). Commercially important sea cucumbers of the world. FAO Species Catalogue for Fishery Purposes, 6, $1-150$.

Semarnap (Secretaría de Medio Ambiente, Recursos. Naturales y Pesca) (1994). Norma Oficial Mexicana NOM-059-ECOL-1994, que determina las especies y subespecies de flora y fauna silvestres terrestres y acuáticas en peligro de extinción, amenazadas, raras y las sujetas a protección especial, y que establece especificaciones para su protección. México: SEGOB.

Semarnat (Secretaría de Medio Ambiente y Recursos Naturales). (2010). Norma Oficial Mexicana NOM-059-ECOL-2010, que determina las especies y subespecies de flora y fauna silvestres terrestres y acuáticas en peligro de extinción, amenazadas, raras y las sujetas a protección especial, y que establece especificaciones para su protección. México, D.F.: SEGOB.

Weisberg, S. (2013). Applied linear regression. Hoboken: Wiley.

Zar, J. H. (2009). Biostatistical analysis. Englewood Cliffs: Prentice-Hall. 